\title{
Bosniak classification of cystic renal masses: utility of contrast- enhanced ultrasound using version 2019
}

\author{
Chau Hung Lee', Joel Jing Kai Liu², Yuxin Zheng², Cher Heng Tan,3 \\ ${ }^{1}$ Department of Radiology, Tan Tock Seng Hospital, ${ }^{2} \mathrm{MOH}$ Holdings, ${ }^{3}$ Lee Kong Chian School of Medicine, Nanyang \\ Technological University, Singapore
}

\begin{abstract}
Aim: To compare the latest 2019 version of Bosniak classification $\left(\mathrm{BC}_{\text {new }}\right)$ against Bosniak classification prior to 2019 $\left(\mathrm{BC}_{\text {old }}\right)$ using contrast-enhanced ultrasound (CEUS) and to compare CEUS against contrast-enhanced CT (CECT) based on $\mathrm{BC}_{\text {new }}$. Material and methods: Patients who had both CEUS and CECT of the kidneys performed within three months of each other were included. CECT and CEUS images of renal cysts were retrospectively analysed by two independent readers using $\mathrm{BC}_{\text {new }}$, extrapolating the $\mathrm{BC}_{\text {new }}$ criteria to CEUS. Where histopathology was not available, 3-year imaging follow-up was used as a reference standard. Results: Forty-nine patients with a total of 54 cysts were included. Using $\mathrm{BC}_{\text {new }}$, Bosniak category between CEUS and CECT and both readers was concordant in 18 cysts (33.3\%). Bosniak category between CEUS and CT was concordant in 27 cysts $(50 \%)$ in reader 1 and in 33 cysts $(61 \%)$ for reader 2 . Based on Cohen's weighted kappa statistic $(\mathrm{k})$, inter-observer agreement was moderate for CEUS $(\mathrm{k}=0.49)$ and fair for CECT $(\mathrm{k}=0.36)$. Agreement between CEUS and CECT for both readers was fair (reader $1, \mathrm{k}=0.24$; reader 2, $\mathrm{k}=0.37$ ). Compared to using $\mathrm{BC}_{\text {old }}$, almost half of the benign cysts were assigned to a lower Bosniak category with CEUS using $\mathrm{BC}_{\text {new }}$ (reader 1, 42.6\%; reader 2, 50\%). Conclusions: CEUS assessment based on $\mathrm{BC}_{\text {new }}$ more appropriately assigns benign renal cysts to a lower category than CEUS based on $\mathrm{BC}_{\text {old }}$. Readers tend to grade renal cysts to a higher Bosniak category with $\mathrm{BC}_{\mathrm{new}}$ but with greater inter-reader agreement on CEUS than on CECT.
\end{abstract}

Keywords: kidney diseases; cyst; Bosniak classification; contrast-enhanced ultrasonography; contrast-enhanced computed tomography

\section{Introduction}

Renal cysts are very common, with an estimated prevalence on imaging of up to $55 \%$ over 70 years old [1]. With an increasing use of imaging, these are often detected incidentally. Most cysts are benign as only an estimated $6 \%$ represent cystic renal cancers [2].

The Bosniak classification was first developed in 1986, with the aim of stratifying the risk of malignancy

Received 22.03.2020 Accepted 05.05.2020

Med Ultrason

2020, Vol. 22, No 3, 279-286

Corresponding author: Chau Hung, Lee

Department of Radiology, Tan Tock Seng Hospital, Singapore,

11 Jalan Tan Tock Seng, Singapore 308433

Phone: +65-6357811

E-mail: chau_hung_lee@ttsh.com.sg in renal cysts. A Bosniak I cyst is almost certainly benign, while a Bosniak IV cyst is almost certainly malignant [3]. The classification scheme was originally established for contrast-enhanced computed tomography (CECT). Apart from inclusion of Bosniak IIF category in 1993, the Bosniak classification has remained largely unchanged since [4]. Over time, the Bosniak classification has been shown to suffer from high inter-observer variability and a large variation in the rate of malignancy, across all Bosniak categories [5-7].

To reduce interobserver variability and improve the precision of reported malignancy rates in the evaluation of complex cystic renal lesions, a recent update in the Bosniak classification was published in August 2019 [8]. The new 2019 version of Bosniak classification $\left(\mathrm{BC}_{\text {new }}\right)$ provides more specific definitions of certain terms from the original Bosniak classification $\left(\mathrm{BC}_{\mathrm{old}}\right)$, such as for 
septations ("thin" vs "thickened", "few" vs "multiple"). $\mathrm{BC}_{\text {new }}$ now disregards ambiguous terms such as "measurable" vs "perceivable" enhancement. Besides CECT, BC${ }_{\text {new }}$ criteria now incorporate contrast-enhanced magnetic resonance imaging (CEMRI) findings.

Given the high spatial resolution of ultrasound and the ability to depict capillary enhancement in real-time, contrast-enhanced ultrasound (CEUS) may be a highly sensitive imaging modality for the evaluation of cystic renal lesions, as an alternative to CECT or CEMRI [9]. Ragel et al found that CEUS allowed for adequate follow-up of complex renal lesions without the drawbacks of CECT (which comes at a higher cost, with ionising radiation and nephrotoxicity of contrast agents). However, the authors also acknowledged the challenge of operator dependence in CEUS [10]. Furthermore, the role of ultrasound (and consequently CEUS) has yet to be fully established [8].

In this study, we sought primarily to determine if the $\mathrm{BC}_{\text {new }}$ when applied to CEUS, more appropriately grades cysts than the $\mathrm{BC}_{\text {old }}$. Interobserver variability, and secondarily, how inter-observer agreement in CEUS would differ from CECT, was also assessed.

\section{Material and methods}

\section{Patient cohort}

This was a single-centre retrospective study approved by the hospital's institutional review board. Power analysis was performed to determine optimal sample size. Based on a similar study, with type I error rate set to 0.05 , odds ratio 0.25 and a statistical power of 0.8 , at least 46 cysts were required [10].

The Radiology department's Picture Archiving and Communication System (PACS) database was searched for all patients who had undergone a renal CEUS study over a 3-year period from January 2014 to December 2016. Inclusion criteria were: (a) subjects with renal cysts evaluated by renal CEUS study, who (b) had an abdominal CECT study performed within 3 months of the CEUS study (to allow meaningful comparison between CEUS and CECT findings) and (c) had at least a 3-year follow-up of the cysts either with ultrasound or cross-sectional imaging following the more recent selected CEUS or CECT study, or had histological correlation through surgical resection or biopsy. Exclusion criteria were: (a) subjects who did not have comparison CECT study available within 3 months or had a CT study performed without intravenous contrast; (b) subjects where the CEUS or CECT study provided a diagnosis apart from a cystic renal mass, specifically if imaging diagnosed a completely solid renal tumour, renal abscess or scar tis- sue; (c) CEUS confirmed a pseudolesion on grey-scale ultrasound; (d) renal cysts that were possibly malignant (significantly increased in size or complexity over 3 years) but did not have histological correlation.

\section{Imaging technique}

All CEUS studies were performed on either a Philips IU22 or Toshiba Alpio 500 ultrasound system, using a curvilinear probe. Mechanical Index ranged from 0.02 to 0.2 . Frequency was fixed at $1.5 \mathrm{MHz}$. Focal zone was placed at the centre of the lesion. For each scan, $2.4 \mathrm{ml}$ of the microbubble contrast agent SonoVue (Bracco, Milan, Italy) was administered as a bolus intravenously via a 22-gauge cannula. Real-time ultrasound cine-clips were acquired in the cortical and parenchymal phase from the start of contrast administration for at least 3 minutes [11].

Contrast-enhanced CT studies were performed on either a Siemens Somatom Definition 64-slice scanner (Siemens Healthineers, Erlangen, Germany) or a Toshiba Aquillon 32-slice scanner (Toshiba Medical Systems, Tochigi, Japan). For each scan, between 80 to $100 \mathrm{ml}$ of Omnipaque 350 (GE Healthcare, Illinois, USA) was administered intravenously via an 18 or 22 -gauge cannula. CECT studies were performed with one of the following protocols: routine abdominopelvic $\mathrm{CT}$ (portovenous phase only), multiphasic renal CT (plain, corticomedullary, nephrographic and execretory phases) or split-bolus CT urography (plain and split-bolus post-contrast phases at 1-min and 8-min). All CT images were reconstructed to $3 \mathrm{~mm}$ thickness. Multiplanar reconstruction in the coronal plane was also performed in the portovenous, nephrohraphic or split-bolus post-contrast phases (depending on protocol).

\section{Image interpretation}

All CEUS and CT studies were reviewed by two readers (one board-certified genitourinary radiologist, C.H.L and one final year senior radiology resident, J.J.K.L, with ten and four years' experience in reading abdominal CT and renal CEUS respectively). The readers familiarised themselves with the $\mathrm{BC}_{\text {new }}$, prior to the review [8]. Each reader independently reviewed the CEUS images, followed by the CECT images in two separate reading sessions at least 1 week apart, in random order. All phases from the CECT studies were available for review. Readers were blinded to the follow-up imaging or histological results at time of review. For lesion grading, CECT criteria for each Bosniak category was extrapolated to CEUS [8]. The $\mathrm{BC}_{\text {new }}$ criteria modified for CEUS is shown in Table I.

\section{Diagnostic reference standard}

Reference standard was histopathological reports from surgical resection or percutaneous biopsy. Where histological correlation was not available, stability of 
Table I. Bosniak categories based on the updated Bosniak classification version 2019, modified for CEUS [8]. The anatomical description for each Bosniak category carefully mirrored the description for CT.

\begin{tabular}{ll}
\hline $\begin{array}{l}\text { Bosniak } \\
\text { category }\end{array}$ & Modified $\mathbf{B C}_{\text {new }}$ description for CEUS \\
\hline I & $\begin{array}{l}\text { Cystic lesions with well-defined, thin }(\leq 2 \mathrm{~mm}) \text { smooth wall, completely anechoic, no septa or calcifications; } \\
\text { wall may enhance }\end{array}$ \\
II & $\begin{array}{l}\text { Cystic lesions with thin }(\leq 2 \mathrm{~mm}) \text { and few }(1-3) \text { septa; septa and wall may enhance; may have calcification or any type } \\
\text { Hypoechoic lesions but without internal enhancement (cyst with echoes) } \\
\text { Cystic lesions that are too small for enhancement to be characterised }\end{array}$ \\
IIF & $\begin{array}{l}\text { Cystic lesions with smooth, minimally thickened }(3 \mathrm{~mm}) \text { enhancing wall or septa } \\
\text { Cystic lesions with many }(\geq 4), \text { smooth thin }(\leq 2 \mathrm{~mm}) \text { enhancing septa }\end{array}$ \\
III & $\begin{array}{l}\text { Cystic lesions with one or more enhancing thick }(\geq 4 \mathrm{~mm}) \text { wall or septa } \\
\text { Cystic lesions with enhancing irregular }(\leq 3 \mathrm{~mm} \text { obtusely-margined convex protrusions) wall or septa }\end{array}$ \\
IV & $\begin{array}{l}\text { Cystic lesions with one or more enhancing nodules }(\geq 4 \mathrm{~mm} \text { obtusely-margined convex protrusions or a convex } \\
\text { protrusion of any size with acute margins })\end{array}$ \\
\hline
\end{tabular}

cysts on imaging in terms of complexity and size (arbitrarily taken as $<25 \%$ increase in maximal diameter) over 3 years was reviewed by the two readers and a third investigator, and assumed to be a sign of benignity [12,13].

Correlation between image interpretation and reference standard

A third investigator, who was not involved in the readings, independently retrieved and recorded the $\mathrm{BC}_{\text {old }}$ of prior CEUS radiology reports as well as the histopathological reports from the hospital electronic medical records. Correlation of image interpretation $\left(\mathrm{BC}_{\text {new }}\right.$ on CEUS and CECT) with $\mathrm{BC}_{\text {old }}$ and reference standards was performed in consensus by all three investigators, at least one week after completion of imaging review of the included CEUS and CT studies, to reduce recall bias.

\section{Statistical analysis}

Inter-observer agreement for each modality in image interpretation, and the agreement between CEUS and CECT, based on BC, were derived using Cohen's weighted kappa statistic (k). Agreement was considered poor if the $\mathrm{k}$-value was less than 0.20 , fair between 0.21 and 0.40 , moderate between 0.41 and 0.60 , good between 0.61 and 0.80 and very good between 0.81 and 1 [14]. Results are presented as the kappa statistic with $95 \%$ confidence intervals.

\section{Results}

\section{Patient cohort}

Over the 3-year period, 64 patients had both CECT and CEUS studies performed within 3 months of each modality. Fifteen patients were excluded for the following reasons: eight patients had CT performed without intravenous contrast, three patients had solid renal masses diagnosed on CEUS, two patients underwent CEUS which did not show a focal renal lesion (i.e. pseudole- sions on grey-scale ultrasound), one patient had a renal abscess confirmed on contrast-enhanced CT, one patient had a Bosniak II cyst that progressed to a Bosniak IIF cyst over the follow-up interval but did not have a histological correlation, therefore could not be ascertained to be benign or malignant.

A total of 49 patients and 54 cysts were thus included in our study (31 males age range was between 39 and 94 years. median 73 years) Nineteen patients had normalmildly impaired renal function (eGFR $>60 \mathrm{ml} / \mathrm{min}$ ), 25 patients had moderate renal impairment (eGFR 30-59 ml/ $\mathrm{min})$ and $5 \mathrm{had}$ severe renal impairment (eGFR $<30 \mathrm{ml} /$ $\mathrm{min}$ ) [15]. The renal cysts ranged in sizes from $0.8 \mathrm{~cm}$ to $10.6 \mathrm{~cm}$ (median $2.1 \mathrm{~cm}$ ). Histopathology was available for four cystic lesions: one was a benign epithelial cyst, one was a chromophobe subtype of renal cell carcinoma (RCC) and two were clear cell subtypes of RCC. All the remaining 50 cysts were followed-up using ultrasound or cross-sectional imaging for a period of at least 3 years and were stable by reference standard definition and assumed to be benign.

Based on the original reports at time of CEUS scan, all CEUS scans had a Bosniak category assigned to the renal cyst of interest, while only 10 out of 54 CT scans had reported a Bosniak category. Reported Bosniak categories can be assumed to be based on $\mathrm{BC}_{\text {old }}$.

The distribution of Bosniak categories using $\mathrm{BC}_{\text {new }}$ by reader and imaging modality is summarised in Tables II and III.

\section{Inter-reader agreement using $B C_{n e w}$}

There was complete agreement in the Bosniak category between both modalities and readers for 18 cysts (33.3\%) using $\mathrm{BC}_{\text {new }}$ (fig 1). There were 38 cysts (70.4\%) given the same Bosniak class by both observers using CEUS and 32 cysts (59.3\%) given the same Bosniak class using CECT. Based on Cohen's kappa statistic (k), 
Table II. Distribution of Bosniak categories based on CEUS by each reader.

\begin{tabular}{llllll}
\hline Bosniak category assigned on CEUS & I & II & IIF & III & IV \\
Reader 1 & $10(18.5)$ & $33(61.1)$ & $5(9.3)$ & $5(9.3)$ & $1(1.9)$ \\
Reader 2 & $31(57.4)$ & $32(59.3)$ & $5(9.3)$ & $4(7.4)$ & $0(0)$ \\
\hline
\end{tabular}

The results are expressed as number (\%).

Table III. Distribution of Bosniak categories based on CT by each reader

\begin{tabular}{llllll}
\hline Bosniak category assigned on CT & I & II & IIF & III & IV \\
\hline Reader 1 & $23(42.6)$ & $22(40.7)$ & $5(9.3)$ & $2(3.7)$ & $2(3.7)$ \\
Reader 2 & $21(38.9)$ & $25(46.3)$ & $5(9.3)$ & $3(5.6)$ & $0(0)$ \\
\hline
\end{tabular}

The results are expressed as number (\%).

there was moderate inter-reader agreement between the two readers for CEUS $[\mathrm{k}=0.49(0.28-0.7)]$ and fair agreement for CECT $[\mathrm{k}=0.36(0.15-0.56)]$ when applying $\mathrm{BC}_{\text {new }}$. A subgroup analysis of Bosniak IIF and III cysts was performed. Out of 8 and 5 cysts assigned a Bosniak IIF on CEUS and CECT respectively, inter-reader agreement was seen in 1 and 3 respectively (12.5\% agreement for CEUS vs $60 \%$ agreement for CECT). Out of 8 and 4 cysts assigned Bosniak III on CEUS and CECT respectively, inter-reader agreement was seen in 1 on both modalities (12.5\% agreement for CEUS vs $25 \%$ agreement for CECT).

Agreement between CEUS and CECT using BC ${ }_{\text {new }}$

Using $\mathrm{BC}_{\text {new }}$ there was agreement in the Bosniak category between CEUS and CECT in 27 cysts $(50 \%)$ for reader 1 , and 33 cysts $(61 \%)$ for reader 2 . CEUS assigned a higher Bosniak category than CECT for readers 1 and 2 in $40.7 \%$ and $27.8 \%$ of cysts respectively, and a lower Bosniak category in $9.3 \%$ and $11.1 \%$ respectively (fig 2). Based on Cohen's kappa statistic (k), there was fair agreement in $\mathrm{BC}$ between CEUS and $\mathrm{CT}$ for both readers [reader 1: $\mathrm{k}=0.24(0.04-0.44)$ and reader 2 : $\mathrm{k}=0.37(0.16$ $0.58)]$.

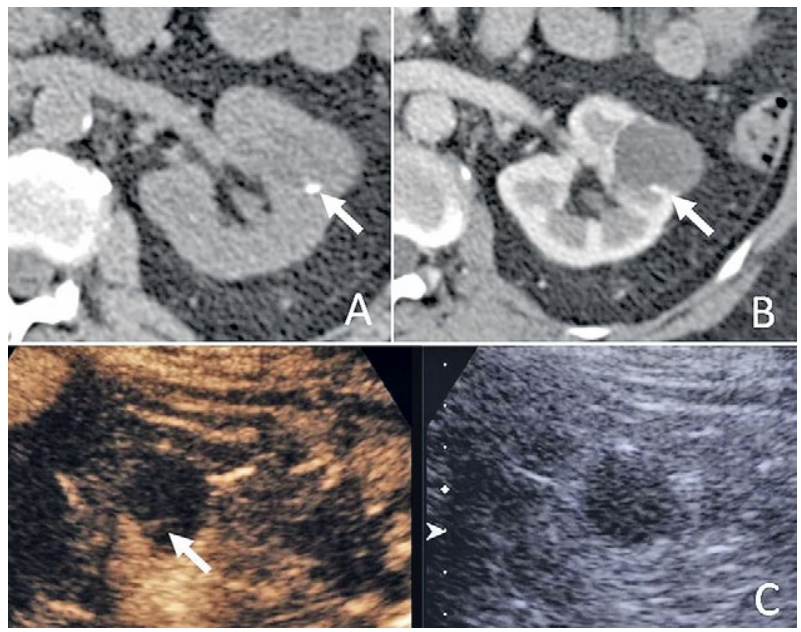

Fig 1. A 61-year-old female had a multiphasic CT of kidneys performed as part of work-up for microscopic hematuria. Plain scan showed a left renal cyst with a thin calcified septation (arrow, A). Corticomedullary phase $\mathrm{CT}$ showed faint enhancement of the thin $1 \mathrm{~mm}$ septation (arrow, B). CEUS was performed as follow-up, which showed a thin $1 \mathrm{~mm}$ enhancing septation (arrow, C). This was categorised as Bosniak II by both readers on both CT and CEUS. It was also categorised as Bosniak II based on $\mathrm{BC}_{\text {old. }}$ The cyst was stable over 3 years.

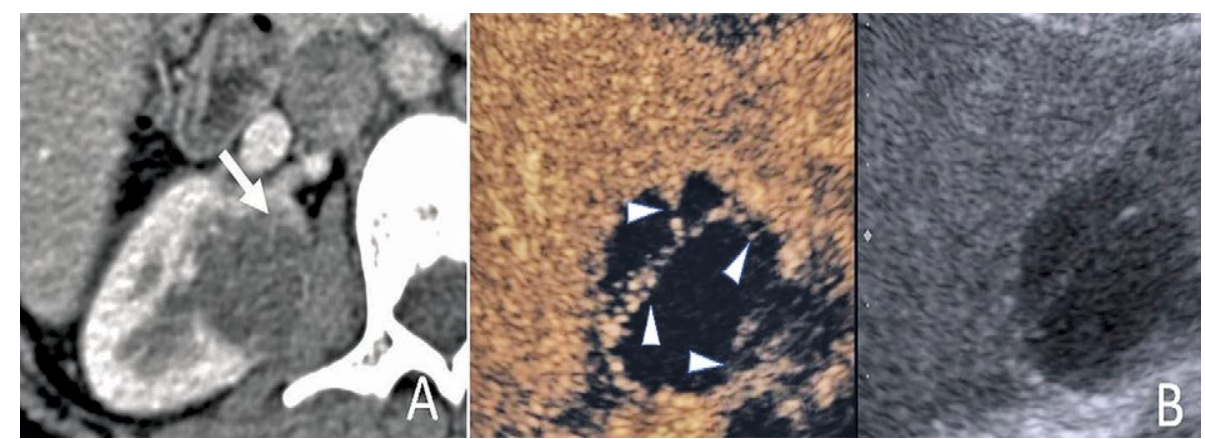

Fig 2. A 49-year-old female had a CECT of the abdomen performed as part of work-up for chronic abdominal discomfort. This showed a left renal cyst with a few $(<4)$ thin 1-2 mm septations (arrow, A). CEUS was performed as a follow-up, which showed multiple (at least 4) thin but partially enhancing septations, demonstrating the higher sensitivity of ultrasound for septations (arrowheads, B). This was categorised as Bosniak IIF on CEUS but Bosniak II on CT by both readers. The cyst was stable over 3 years and assumed to be benign. This was reported as Bosniak III on CEUS using $\mathrm{BC}_{\text {old }}$, and highlights how the less ambiguous definitions in $\mathrm{BC}_{\text {new }}$ allowed for the appropriate downgrading of the Bosniak category for this cyst. 
Comparison between $B C_{\text {new }}$ and $B C_{\text {old }}$ using $C E U S$

For this analysis, the reports of the CEUS studies were reviewed for the Bosniak category at time of reporting (based on $\mathrm{BC}_{\text {old }}$ ). All $54(100 \%)$ cysts had been assigned a Bosniak category based on $\mathrm{BC}_{\text {old }}$ in the radiology reports. The Bosniak category based on $\mathrm{BC}_{\text {new }}$ was obtained through independent reviews by the 2 readers for this study. When compared to the $\mathrm{BC}_{\mathrm{old}}$, readers 1 and 2 assigned $51.9 \%$ and $46.3 \%$ of cysts into the same category, $42.6 \%$ and $50 \%$ of cysts were assigned a lower category, while $5.6 \%$ and $3.7 \%$ of cysts were assigned a higher category.

Of a total of 31 cysts assigned a lower Bosniak category by both readers on CEUS based on $\mathrm{BC}_{\text {new }}$ compared to $\mathrm{BC}_{\text {old }}, 30$ were stable in terms of size and complexity and were assumed to be benign. One was histologicallyproven to be malignant.
Four cysts had a histological correlation: (1) A $6 \mathrm{~cm}$ complex cystic lesion was graded as Bosniak IIF by both readers on CEUS. This was reported as Bosniak III based on $\mathrm{BC}_{\text {old }}$. On CECT, this was graded as Bosniak IV and III by reader 1 and 2 respectively. Nephrectomy confirmed renal cell carcinoma (fig 3). (2) A $6 \mathrm{~cm}$ complex cystic was graded Bosniak IV and III by reader 1 and 2 respectively on CEUS. This was reported as Bosniak III based on $\mathrm{BC}_{\text {old }}$. On CECT, this was graded as Bosniak II and III by reader 1 and 2 respectively. Nephrectomy confirmed chromophobe RCC (fig 4). (3) A 2 cm cystic lesion was graded Bosniak II and I by reader 1 and 2 respectively on CEUS. This was reported as Bosniak I based on $\mathrm{BC}_{\text {old }}$. On CECT, this was graded as Bosniak III by both readers. A follow-up CECT after 2 years showed size increase and new nodular irregular mural thickening (progression to Bosniak IV). Nephrectomy confirmed clear cell RCC
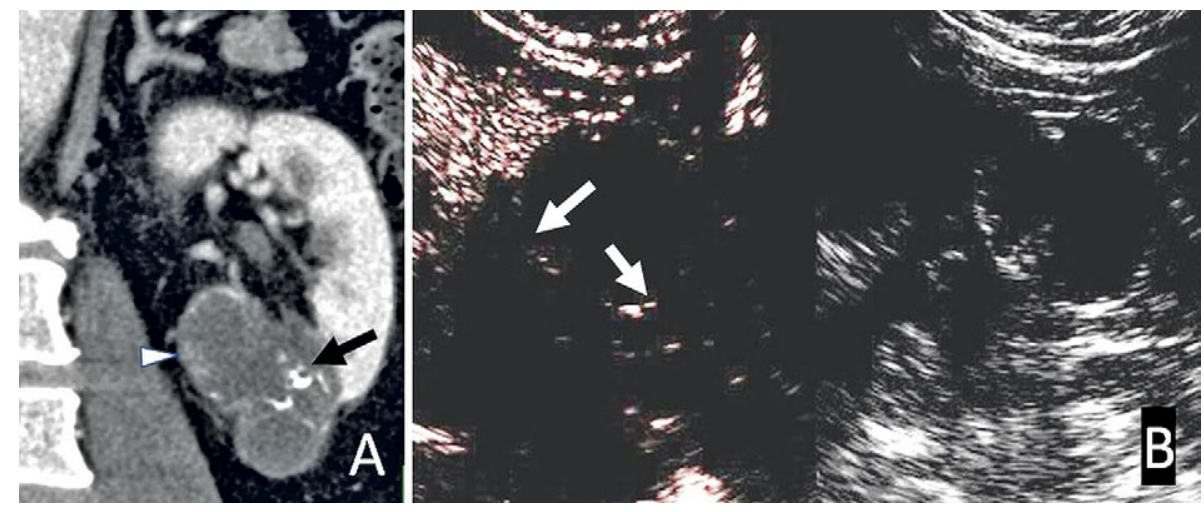

Fig 3. A 52-year-old male had a CT of the kidneys performed as part of work-up for microscopic hematuria. Coronal reformat in the nephrographic phase showed a complex left renal cyst with calcified, thick (at least $4 \mathrm{~mm}$ ) enhancing septations (arrow, A) with possible enhancing nodules (arrowhead, A). This was categorised as Bosniak IV and Bosniak III by reader 1 and 2 respectively on CECT. In the corresponding CEUS, only multiple septations but not the eccentrically-located mural nodules within the cystic lesion were visualised, highlighting how a large lesion size may make it difficult for complete lesion interrogation (arrow, B). This was categorised as Bosniak IIF by both readers on CEUS and reported Bosniak III based on $\mathrm{BC}_{\mathrm{old}}$. Nephrectomy confirmed clear cell RCC.


Fig 4. A 77-year-old male had ultrasound performed as part of work-up for microscopic hematuria which showed a cyst with internal echoes in the right kidney (not shown). CEUS was performed for further evaluation which showed a cyst with multiple thick septations and possible enhancing mural nodules (arrow, A). This was categorised as Bosniak IV and Bosniak III by reader 1 and 2 respectively, and reported as Bosniak III based on $\mathrm{BC}_{\text {old }}$. Coronal reformatted image of the single phase portovenous phase CECT showed a cystic lesion with a few thin 1-2 mm enhancing septations (arrow, B). This was categorised as Bosniak II and Bosniak III by reader 1 and 2 respectively, illustrating how enhancing mural nodules are better visualised on CEUS compared to CECT. Nephrectomy confirmed chromophobe subtype of RCC. 

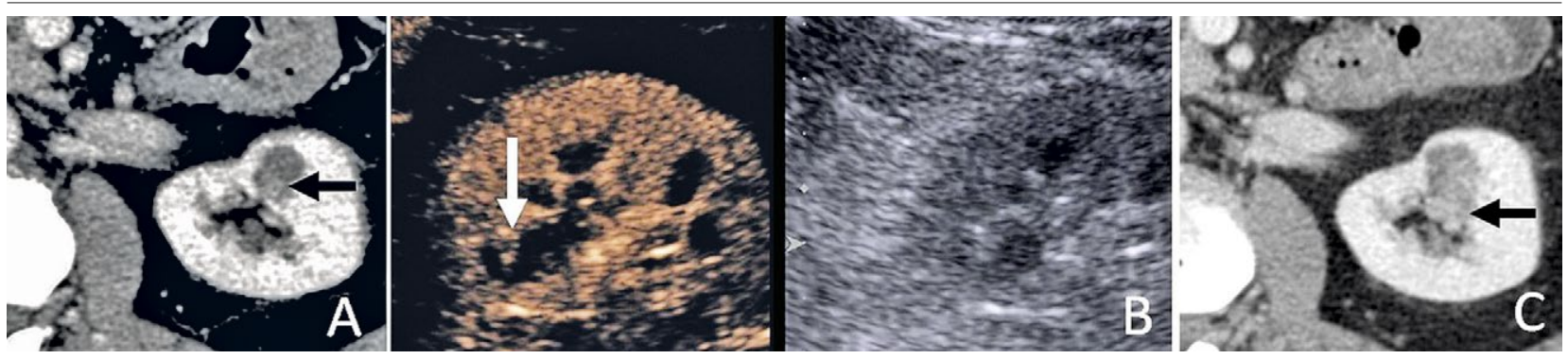

Fig 5. A 56-year-old gentleman had a CECT scan performed for epigastric discomfort. A complex cystic lesion with eccentric 5 mm thick enhancing wall was seen in the left kidney (arrow, A). This was categorised as Bosniak III by both readers on CECT. (B) CEUS performed as follow-up, showed a cyst with a thin $2 \mathrm{~mm}$ faintly enhancing septation (arrow, B). This was categorised as Bosniak II and I on CEUS by readers 1 and 2 respectively and reported as Bosniak I based on $\mathrm{BC}_{\text {old }}$. This highlights the limitations of CEUS in terms of operator-dependence. The patient was lost to follow-up. Two years later, a repeat CECT scan showed the cystic lesion to have increased in size with more prominent mural nodules suspicious for a Bosniak IV cyst (arrow, C). There was no corresponding CEUS for the repeat CECT scan. Nephrectomy confirmed clear cell RCC.

(fig 5). (4) A $4 \mathrm{~cm}$ cystic lesion graded as Bosniak III by both readers on CEUS. This was also reported as Bosniak III based on $\mathrm{BC}_{\text {old }}$. On CECT, this was graded as Bosniak IIF and III by reader 1 and 2 respectively (fig 6). Biopsy was negative for malignancy.

\section{Discussions}

The version 2019 of the Bosniak classification of cystic renal masses $\left(\mathrm{BC}_{\text {new }}\right)$ now provides users with more specific definitions of the lexicon for each Bosniak category [8]. For example, it defines "thin" $(\leq 2 \mathrm{~mm})$, "minimally thickened" (3 mm) and "thickened" $(\geq 4$ $\mathrm{mm})$ septations, and "few" (1-3) vs "multiple" (>3) septations. This allows a more objective Bosniak classification of renal cysts.

$\mathrm{BC}_{\text {new }}$ applies to contrast-enhanced $\mathrm{CT}$ and MRI. Although the role of ultrasound is not fully established, CEUS can be potentially useful in the evaluation of complex cystic renal lesions [16,17]. Moreover, CEUS is particularly attractive due to its lower cost, lack of ionizing radiation and non-nephrotoxic microbubble contrast agents. Studies have shown that CEUS is able to demonstrate enhancing nodules and thickened septations better than CT, and CEUS classified most cysts as either the same or higher Bosniak category compared to CT, although this does not seem to lead to a significantly better diagnostic accuracy [18-22].

In our study comprising mostly benign cysts, up to $40 \%$ of cysts are assigned a higher Bosniak category on CEUS compared to CECT based on $\mathrm{BC}_{\text {new }}$. This is not unexpected given that septations and small nodules are better seen on ultrasound and is consistent with other publications on this subject $[23,24]$. In our study we compared readers of different levels of clinical experience and expertise but found only fair agreement in the
Bosniak classification between CEUS and CECT, even for the more experienced reader. When CEUS findings suggest a Bosniak III or IV cystic lesion, it would therefore be prudent to correlate with contrast-enhanced CT or MRI, if surgical intervention is being considered. Despite
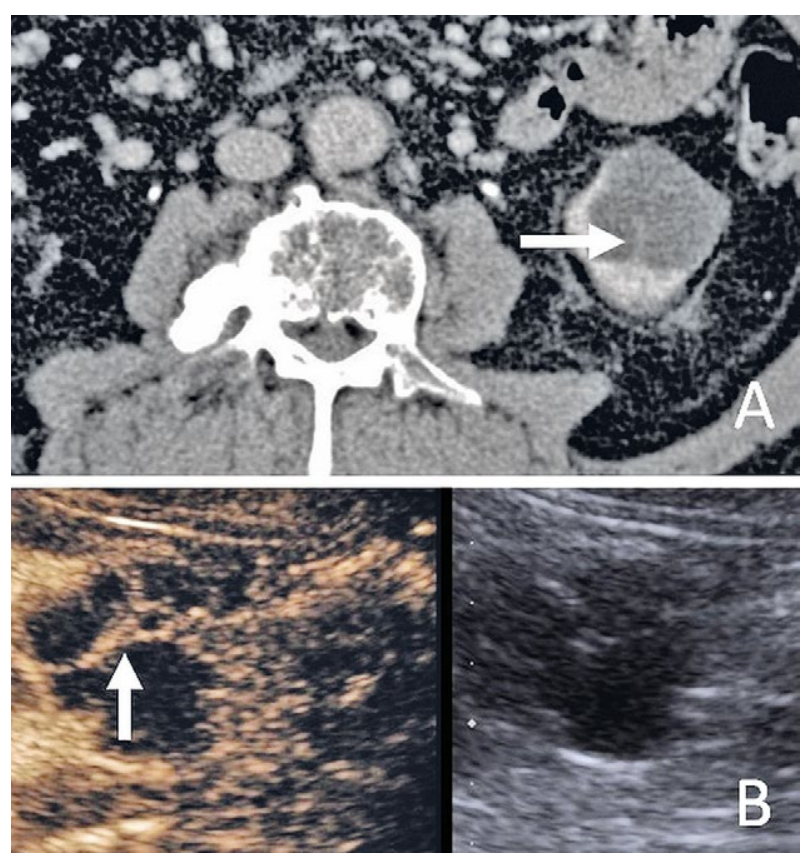

Fig 6. A 73-year-old male had CECT scan performed for workup to exclude abdominal malignancy. Axial CECT shows a septated cyst in the left kidney with at least one of the septations (arrow) appearing minimally thickened or thick, measuring about 3-4 mm think (arrow, A). This was graded Bosniak IIF and Bosniak III on CT by readers 1 and 2 respectively. CEUS was performed as follow-up, which showed thick (4 $\mathrm{mm}$ ) enhancing septations (arrow, B). This was graded Bosniak III on CEUS by both readers, and also reported as Bosniak III based on $\mathrm{BC}_{\text {old }}$. Needle biopsy of the septations was negative for malignancy. 
CEUS tending to assign a high Bosniak category compared to CECT, for the three histologically-proven malignancies in our study, CEUS under-graded the Bosniak category for two lesions compared to CECT and appropriately upgraded the Bosniak category for one lesion, while $\mathrm{BC}_{\text {new }}$ under-graded one lesion compared to $\mathrm{BC}_{\text {old }}$ on CEUS (Bosniak IIF using $\mathrm{BC}_{\text {new }}$ vs Bosniak III using $\left.\mathrm{BC}_{\text {old }}\right)$. The statistical significance of this observation is not known, due to the small number of lesions that were proven malignant ( 3 out of $54,5.6 \%$ ). When we retrospectively reviewed these cases, it is likely that the most suspicious components in these lesions were obscured or not fully interrogated at the time of the CEUS study. This highlights the limitations of CEUS attributable to operator-dependence and patient factors.

Our results showed that applying $\mathrm{BC}_{\text {new }}$ on CEUS allowed appropriate reclassification of up to half of the benign cysts to a lower Bosniak category (compared to $\mathrm{BC}_{\mathrm{old}}$ ), potentially reducing unnecessary follow-up or intervention for patients. We attributed this to more specific definitions in the updated Bosniak classification allowing for more objective grading.

Interestingly, our study found a higher overall interreader agreement for CEUS compared to CECT, when applying $\mathrm{BC}_{\text {new }}$. Interobserver agreement in prior studies applying $\mathrm{BC}_{\text {old }}$ to either CECT or CEUS have been variable, ranging from poor to very good $[18-22,25]$. The largest degree of disagreement tends to be between the Bosniak IIF and III complex cysts [26,27]. For the subgroup of Bosniak IIF and III cysts in our cohort, CEUS demonstrated a lower inter-reader agreement for Bosniak IIF and III cysts compared to CECT. While this may be due to the higher sensitivity of ultrasound for nodules and septations resulting in a potential for over-grading, we also believe that reader experience might play an important role in assessing the more complex features such as borderline septal thickening and subtle nodules. The differences in reader experience and level of expertise in our study could potentially have under-estimated the inter-reader agreement [28]. However, our study had too few proven malignant lesions for further analysis in this aspect. It has been hypothesized that more specific definitions in $\mathrm{BC}_{\text {new }}$ should improve interobserver agreement; however, this remains to be seen [8]. Larger scale prospective studies will be required to further validate the $\mathrm{BC}_{\text {new }}$ in this aspect.

Our study has limitations mainly related to its retrospective nature. For example, different operators performed CEUS. However, this is mitigated as only a few sonographers and radiologists with credentialed experience in CEUS are allowed to operate. In our institution, dynamic images of CEUS images are stored as video clips; this may have reduced potential error during imaging review. Furthermore, the CEUS and CECT scans were not performed concurrently on the same day. We addressed this by only including subjects with both imaging modalities performed within 3 months. The relatively small sample size in our study may also have impeded statistical analysis and limited clinical applicability. Also, we did not re-assess the renal cysts using $\mathrm{BC}_{\text {old }}$ criteria but extracted this from the study reports. This was done as it would be practically difficult for readers to switch between the $\mathrm{BC}_{\text {new }}$ and $\mathrm{BC}_{\text {old }}$ criteria consciously during imaging review. Finally, very few cysts had histological confirmation of final diagnosis, and even fewer lesions were confirmed to be malignant ( 3 out of 54 cysts). Unfortunately, it would not be ethical to subject every patient with a lower Bosniak category to resection or even needle biopsy. As a surrogate marker of benignity, we used a three-year follow-up on imaging, and excluded those that changed in morphology or size without definitive diagnosis.

\section{Conclusions}

More appropriate grading of $\mathrm{BC}_{\text {new }}$ compared to $\mathrm{BC}_{\text {old }}$ for benign renal cysts using CEUS potentially reduces unnecessary interventions or follow-up. $\mathrm{BC}_{\text {new }}$ when applied to CEUS evaluation of renal cysts demonstrates a higher inter-reader agreement compared to CECT, although the inter-reader agreement for Bosniak IIF and III lesions appear lower for CEUS compared to CT. The complete role of CEUS requires further validation with large prospective studies, given that there is only fair agreement with CECT.

\section{Conflict of interest: none}

\section{References}

1. Mensel B, Kühn JP, Kracht F, et al. Prevalence of renal cysts and association with risk factors in a general population: an MRI-based study. Abdom Radiol (NY) 2018;43:30683074.

2. Warren KS, McFarlane J. The Bosniak classification of renal cystic masses. BJU Int 2005;95:939-942.

3. Bosniak MA. The current radiological approach to renal cysts. Radiology 1986;158:1-10.

4. Smith AD, Allen BC, Sanyal R, et al. Outcomes and complications related to the management of Bosniak cystic renal lesions. AJR Am J Roentgenol 2015;204:W550-W556.

5. Schoots IG, Zaccai K, Hunink MG, Verhagen PCMS. Bosniak classification for complex renal cysts reevaluated: a systematic review. J Urol 2017;198:12-21.

6. Sevcenco S, Spick C, Helbich TH, et al. Malignancy rates and diagnostic performance of the Bosniak classification 
for the diagnosis of cystic renal lesions in computed tomography: a systematic review and meta-analysis. Eur Radiol 2017;27:2239-2247.

7. Smith AD, Carson JD, Sirous R, et al. Active surveillance versus nephronsparing surgery for a Bosniak IIF or III renal cyst: a cost-effectiveness analysis. AJR Am J Roentgenol 2019;212:830-838.

8. Silverman SG, Pedrosa I, Ellis JH, et al. Bosniak Classification of Cystic Renal Masses, Version 2019: An Update Proposal and Needs Assessment. Radiology 2019;292:475488.

9. Harvey CJ, Alsafi A, Kuzmich S, et al. Role of US Contrast Agents in the Assessment of Indeterminate Solid and Cystic Lesions in Native and Transplant Kidneys. Radiographics 2015;35:1419-1430.

10. Ragel M, Nedumaran A, Makowska-Webb J. Prospective comparison of use of contrast-enhanced ultrasound and contrast-enhanced computed tomography in the Bosniak classification of complex renal cysts. Ultrasound 2016;24:6-16.

11. Sidhu PS, Cantisani V, Dietrich CF, et al. The EFSUMB Guidelines and Recommendations for the Clinical Practice of Contrast-Enhanced Ultrasound (CEUS) in Non-Hepatic Applications: Update 2017 (Short Version). Ultraschall Med 2018;39:154-180.

12. Zhong J, Cao F, Guan X, Chen J, Ding Z, Zhang M. Renal cyst masses (Bosniak category II-III) may be over evaluated by the Bosniak criteria based on MR findings. Medicine (Baltimore) 2017;96:e9361.

13. Herts BR, Silverman SG, Hindman NM, et al. Management of the incidental renal mass on CT: A white paper of the ACR incidental findings committee. J Am Coll Radiol 2018;15:264-273.

14. Landis JR, Koch GG. The measurement of observer agreement for categorical data. Biometrics 1977;33:159-174.

15. 15 Stevens PE, Levin A, Kidney Disease: Improving Global Outcomes Chronic Kidney Disease Guideline Development Work Group Members. Evaluation and Management of Chronic Kidney Disease: Synopsis of the Kidney Disease: Improving Global Outcomes 2012 Clinical Practice Guideline. Ann Intern Med. 2013;158:825830.

16. Shahzad K, Simms MS, Byass O. Evaluation of contrast enhanced ultrasound for investigation of complex cystic renal masses. J Clin Urol 2011;4:253-258.
17. Öztürk H. Evaluation of Bosniak Type IIF and III Renal Cysts with Contrast-enhanced Ultrasound. J Belg Soc Radiol 2016;100:12.

18. Sanz E, Hevia V, Gomez V, et al. Renal Complex Cystic Masses: Usefulness of Contrast-Enhanced Ultrasound (CEUS) in Their Assessment and Its Agreement with Computed Tomography. Curr Urol Rep 2016;17:89.

19. Park BK, Kim B, Kim SH, Ko K, Lee HM, Choi HY. Assessment of cystic renal masses based on Bosniak classification: comparison of CT and contrast-enhanced US. Eur J Radiol 2007;61:310-314.

20. Xue LY, Lu Q, Huang BJ, et al. Contrast-enhanced ultrasonography for evaluation of cystic renal mass: in comparison to contrast-enhanced CT and conventional ultrasound. Abdom Imaging 2014;39:1274-1283.

21. Quaia E, Bertolotto M, Cioffi V, et al. Comparison of contrast-enhanced sonography with unenhanced sonography and contrast-enhanced CT in the diagnosis of malignancy in complex cystic renal masses. AJR Am J Roentgenol 2008;191:1239-1249.

22. Ascenti G, Mazziotti S, Zimbaro G, et al. Complex cystic renal masses: characterizationwith contrast-enhanced US. Radiology 2007;243:158-165.

23. Hélénon O, Crosnier A, Verkarre V, Merran S, Méjean A, Correas JM. Simple and complex renal cysts in adults: Classification system for renal cystic masses. Diagn Interv Imaging 2018;99:189-218.

24. Clevert DA, Minaifar N, Weckbach S, et al. Multislice computed tomography versus contrast-enhanced ultrasound in evaluation of complex cystic renal masses using the Bosniak classification system. Clin Hemorheol Microcirc 2008;39:171-178.

25. Siegel CL, McFarland EG, Brink JA, Fisher AJ, Humphrey P, Heiken J P. CT of cystic renal masses: analysis of diagnostic performance and interobserver variation. AJR Am J Roentgenol 1997;169:813-818.

26. Graumann O, Osther SS, Karstoft J, Horlyck A, Osther PJ. Bosniak classification system: inter-observer and intra-observer agreement among experienced uroradiologists. Acta Radiol 2015;56:374-383.

27. Smith AD, Remer EM, Cox KL, et al. Bosniak category IIF and III cystic renal lesions: outcomes and associations. Radiology 2012;262:152-160.

28. Crewson PE. Reader agreement studies. AJR Am J Roentgenol 2005;184:1391-1397. 